\title{
La circulation des voitures sur une route ou dans les artères d'une ville, à partir des lois de l'hydraulique
}

\author{
The fluid mechanics approach to town \\ and country traffic flows
}

\author{
PAR R. FORTET, \\ PROFESSEUR A L'INSTITUT HENRI-POINGARÉ, PARIS
}

\begin{abstract}
L'auteur donne la liste des publications, assez peu nombreuses, qui étudient le trafic des véhicules en s'inspirant des méthodes de la Mécanique des fluides; puis il analyse les principales d'entre elles, réparties en deux groupes: a) La théorie des ondes cinématiques de Lighthill et Whitham et ses applications aux «goulots d'etranglement »;

b) La relation flux-densité dite «de Greenberg» pour un trafic de convoi en régime stationnaire, et son établissement $\dot{a}$ partir de lois analogues à celles de l'hydrodynamique;
\end{abstract}

\section{1. - PREAMBULE}

L'étude mathématique du trafic des véhicules ne s'est véritablement développée que depuis environ 1955; cependant, des travaux, qu'on peut considérer comme des travaux de pionniers ont été publiés, un peu sporadiquement, à partir de 1935. Comme il était naturel, beaucoup des premiers chercheurs se sont inspirés de l'analogie avec l'écoulement d'un fluide continu; parmi les articles qui se sont référés à ce point de vue, les principaux, jusqu'à présent, sont les suivants (par ordre chronologique) :

[1] M. J. Lighthill et G. B. Whitham. - On kinematic waves, II; a theory of traffic flow on long crowded roads; Proc, Roy. Soc., A, 229, 1955 , p. 317.

[2] P. I. Richards. - Shock waves on the highway; Oper. Res., 4, 1956, p. 42.

[3] S. C. DE. - Kinematic wave theory of bottlenecks of varying capacity; Proc. Camb. Phil. Soc., 52, 1956, p. 564.
The comparatively few publications which have so far appeared on this subject are listed and the most important analysed. Their subject matter falls within the lwo following groups:-

a) Lighthill and Whitham's kinematic wave theory and its applications to traffic bottlenecks;

b) Greenberg's "flux/density relationship" for continuous traffic streams, and its denelopment. from laws similar to those of hydrodynamics;

[4] M. J. Lighthill. - Dynamic instability of transport systems: the hydrodynamic analogy; Oper. Res. Quat., 8, 1957.

[5] H. Greenberg. -- An analysis of traffic flow; Oper. Res., 7, 1959, p. 79.

[6] J. H. Bick et G. F. NewerL. - A continuum model for two-directional traffic flow: Quart. Appl. Math., 18, 1960, p. 191.

Deux ouvrages sur le trafic des véhicules ont paru récemment; ce sont :

[7] R. Hermann. - Theory of traffic flow; Elsevier edit., New York, 1961.

[8] F. A. Haight. - Mathematical theories of traffic flow; Academic Press, New York, 1963.

[7] est un recueil de conférences prononcées, à l'occasion d'un symposium, par divers auteurs sur des problèmes divers; ni [7] ni [8] ne sont consacrés spécialement à l'analogie hydrodynamique, mais ils s'y réfèrent en certains de leurs passages, sans d'ailleurs apporter rien d'essen. 
tiellement nouveau par rapport à [1], [2], [3], $[4],[5],[6]$.

On comprend immédiatement que l'analogie avec l'hydrodynamique ne peut être pertinente que dans le cas d'un trafic assez dense : un trafic de véhicules en effet est essentiellement constitué d'éléments discrets, les divers couples (véhicule, conducteur). C'est ici l'occasion de préciser la terminologie et les notations que j'emploierai.

En principe, je laisserai de côté les problèmes posés par l'intersection de plusieurs voies; il s'agira done du trafic sur une voie donnée (tronçon déterminée d'une route déterminée, ouvrage d'art, etc.). Observons qu'une même voie donne ordinairement lieu à deux trafics, l'un dans un sens, l'autre en sens inverse; ces deux trafics sont considérés comme distincts; mais, bien entendu, ils peuvent avoir entre eux quelque interaction.

Pour être bref, je parlerai en général uniquement de véhicules, mais il ne faut jamais oublier que les éléments d'un trafic sont des couples (véhicule, conducteur). Les véhicules d'un trafic peuvent être individualisés, par exemple en les numérotant; on peut appeler $x_{n}(t)$ l'abscisse à l'instant $t$, sur la voie assimilée à une courbe géométrique $x^{\prime} \mathrm{O} x$, du véhicule $\mathrm{n}^{\circ} n$; étant entendu que le trafic étudié est celui qui s'écoule en direction des $x$ croissants. Usuellement, la courbe $x^{\prime} \mathrm{O} x$ sera assimilable à un axe rectiligne $x^{\prime} \mathrm{O} x$, et ceci est admis dans la suite. Je précise - cette précision est utile parce que les dimensions d'un véhicule entrent parfois en ligne de compte, - que $x_{n}(t)$ est exactement l'abscisse du pare-chocs avant du véhicule $n^{\circ} n$.

L'étude d'un trafic, surtout si l'on veut s'inspirer de la Mécanique des fluides, suggère d'introduire, pour chaque instant $t$ et chaque abscisse $x$, les notions suivantes:

$$
\begin{aligned}
\varphi=\varphi(t, x): & \text { flux, à l'instant } t \text { et à l'abscisse } x, \\
\rho=\rho(t, x): & \text { densité (ou concentration), à } \\
& \text { l'instant } t \text { et à l'abscisse } x, \\
u=u(t, x): & \text { vitesse à l'instant } t, \text { du véhicule, } \\
& \text { situé à l'instant } t \text { à l'abscisse } x .
\end{aligned}
$$

Pour un trafic assez dense, l'introduction du flux $\varphi$ et de la densité $\rho$ ne soulèvent pas de difficulté sérieuse; par exemple, soit $\mathrm{N}_{1}(t, x ; \Delta t)$ le nombre des véhicules dont le pare-choc avant franchit l'abscisse $x$ entre les instants $(t--\Delta t)$ et $(t+\Delta t)$; il serait absurde d'envisager de faire tendre $\Delta t$ vers +0 ; mais il est clair qu'en choisissant $\Delta t$ raisonnablement petit, le rapport :

$$
\frac{\mathrm{N}_{1}(t, x ; \Delta t)}{2 \Delta t}
$$

fournit une interprétation concrète, une mesure et même une définition valables de $\varphi(t, x)$. De même, en choisissant $\Delta x(\Delta x>0)$, non pas infi- niment petit, mais raisonnablement petit, et en appelant $\mathrm{N}_{2}(t, x ; \Delta x)$ le nombre des véhicules dont le pare-choc avant est à l'instant $t$ situé entre les abscisses $(x-\Delta x)$ et $(x+\Delta x)$, le rapport :

$$
\frac{\mathrm{N}_{2}(t, x ; \Delta x)}{2 \Delta x}
$$

constitue une interprétation concrète, une mesure et même une définition valables de $\rho(t, x)$.

Pour $u$, la situation est plus complexe; $u$ doit être la vitesse des véhicules situés, à l'instant $t$, à l'abscisse $x$; il peut fort bien arriver - cela se produit en particulier en cas de dépassement qu'il $\mathrm{y}$ ait, à l'instant $t$ ou au voisinage de cet instant, plusieurs véhicules situés à l'abscisse $x$ ou dans son voisinage immédiat; et ces divers véhicules peuvent naturellement posséder des vitesses très différentes : c'est évidemment le cas pour un dépassement.

Accepter d'introduire la vitesse $u=u(t, x)$ est une hypothèse, que j'appellerai l'hypothèse d'une vitesse localisée (sous-entendu : dans le temps et dans l'espace); cette hypothèse implique que le trafic étudié ne comporte pas de dépassement (ou du moins que ceux-ci sont assez rares pour ne pas avoir d'influence sensible).

Tous les travaux cités ci-dessus font, généralement sans l'exprimer, l'hypothèse d'une vitesse localisée; certains de leurs résultats sont indépendants de cette hypothèse; quant à ceux qui en dépendent, leur portée est limitée aux trafics sans dépassements. Il est regrettable que les travaux en question n'en aient pas fait clairement la remarque.

[1], après avoir justifié $\varphi$ et $\rho$ à peu près comme je l'ai fait ci-dessus, pose par définition que :

$$
u(t, x)=\frac{\varphi(t, x)}{\rho(t, x)},
$$

ce qui au premier abord semble éliminer toute difficulté pour la définition de $u$; on a évidemment toujours le droit de désigner par la notation $u$ le rapport $\varphi / \rho$; mais le $u$ ainsi obtenu n'est qu'une sorte de vitesse «moyenne», comme [1] le remarque d'ailleurs; après quoi, et sans explication, il semble traiter et interpréter ce $u$, lorsqu'il s'en sert, comme une vitesse réelle de véhicules réels, à laquelle on aurait le droit d'appliquer les principes usuels de la Mécanique; or, ceci n'est justifié que si on admet l'hypothèse d'une vitesse localisée.

L'Éguation de continuté. - Ceci dit, le modèle mathématique du mouvement (unidimensionnel) d'un fluide est constitué par trois équations :

$\alpha=$ équation de continuité;

$\beta=$ équation de la dynamique;

$r=$ équation d'état. 
Il est clair que l'équation de continuité est parfaitement valable pour un trafic (assez dense) de véhicules, et s'écrit :

$$
\frac{\partial p}{\partial t}+\frac{\partial \varphi}{\partial x}=0
$$

Si l'on fait l'hypothèse d'une vitesse localisée, ou encore si on définit $u$ par $(1,1)$, on a :

$$
\varphi=p u \text {, }
$$

et alors $(1,2)$ peut s'écrire :

$$
\frac{\partial \rho}{\partial t}+\frac{\partial}{\partial x}(\rho u)=0 .
$$

\section{2. - LA RELATION FLUX-DENSITE}

On n'aperçoit guère au premier abord ce qui, dans le cas d'un trafic de véhicules, pourrait jouer le rôle de l'équation de la dynamique et de l'équation d'état. Beaucoup de chercheurs ont utilisé pour cela la relation flux-densité.

Les ingénieurs chargés des questions de trafic ont souvent observé que tous les trafics s'écoulant sur une voie donnée et de même densitè $\rho$, donnent lieu au même flux $\varphi$; autrement dit, pour une voie donnée, il y a relation déterminée

$$
\varphi=\mathrm{G}(\mathrm{p})
$$

entre la densité et le flux; la fonction $G$ est une caractéristique de la voie; elle n'est pas nécessairement la même pour toutes les voies, encore qu'on puisse s'attendre à ce qu'elle ait des formes très analogues pour des voies comparables.

\section{Notons que :}

$1^{\circ}$ L'observation en question des ingénieurs est contrôlable par l'expérience, qui peut également fournir une estimation empirique de G;

$2^{\circ}$ A priori, on peut affirmer certaines propriétés de G; il est clair que :

$$
\mathrm{G}(0)=0 \text {; }
$$

il est clair également qu'il existe une valeur positive $\rho_{c}$ de $\rho$, dite densité de congestion, pour laquelle les véhicules du trafic sont tellement englués les uns avec les autres qu'ils ne peuvent plus bouger, de sorte que leur vitesse $u_{c}=0$ et que le flux $\varphi_{c}=0$, autrement dit :

$$
\begin{aligned}
& \varphi_{c}=\mathrm{G}\left(\rho_{c}\right)=0, \\
& u_{c}=0 .
\end{aligned}
$$

$G(p)$ n'est définie par conséquent que sur l'intervalle $\left[0, \rho_{o}\right]$, et il doit exister une valeur $\rho_{m}$ de $\rho$, entre 0 et $\rho_{c}$ pour laquelle $\varphi$ prend sa valeur maximale $\varphi_{m}$ :

$$
\varphi_{m}=\mathrm{G}\left(\rho_{m}\right) \text {. }
$$

On peut donc s'attendre à ce que le graphique de $\mathbf{G}$ ait en général l'allure suivante :

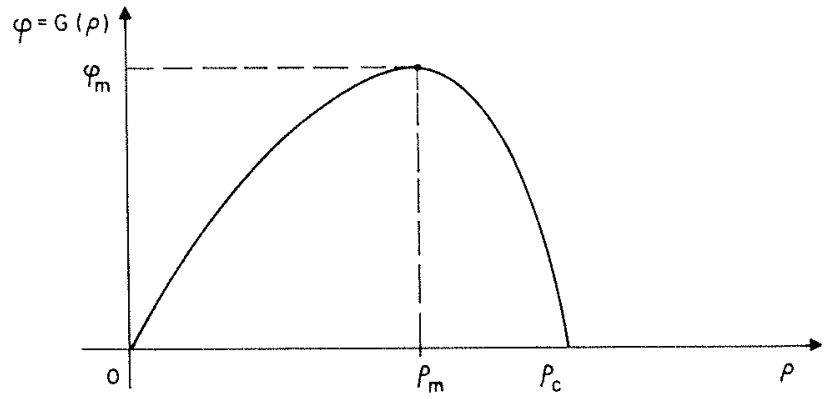

FIG. 2.1

Ceci étant, certains chercheurs ([1], [2], [3], [4], [6]) ont pris le parti de considérer la fonction $\mathrm{G}(\rho)$ comme donnée, la relation $(2,1)$ comme une loi physique, bien établie quoique seulement empiriquement, et valable pour tout trafic; ils adjoignent alors $(2,1)$ à $(1,2)$ pour déterminer mathématiquement le trafic. Leur méthode sera désignée sous le nom de méthode de la relation flux-densité.

Ce faisant, ils n'ont pas porté suffisamment d'attention aux points suivants :

L'observation empirique des ingénieurs aboutissant à $(2,1)$ ne concerne évidemment que des trafics en régime stationnaire; d'abord, parce qu'un trafic en régime rapidement variable est difficile à observer avec précision; ensuite, parce que l'existence d'une relation telle que $(2,1)$ est inconcevable en régime rapidement variable, alors qu'elle est intuitive en régime stationnaire.

Admettre, comme l'ont fait les chercheurs auxquels je fais allusion actuellement, que $(2,1)$ est valable pour un trafic quelconque, est une hypothèse, certainement fausse en régime rapidement variable, mais il est vrai plausible dans le cas de trafics résultant d'un trafic stationnaire faiblement perturbé. Ceci limite nettement la portée de leurs résultats. Surtout si l'on tient compte de la remarque suivante :

Un trafic réel n'adopte un régime stationnaire que sous certaines conditions, assez souvent réalisées en pratique, mais néanmoins restrictives, et qu'on peut résumer en la propriété de stabilité suivante. Propriété de stabilité : La propriété de stabilité consiste en ce que les conditions générales sous lesquelles s'effectue le trafic : propriétés physiques permanentes et occasionnelles de la voie, manière de conduire des conducteurs, taux d'arrivée des véhicules, réglementation policière, ctc., restent stables sur une longueur suffisante de la voie, et pendant une durée de temps suffisante.

A ce propos, je rappelle la définition suivante:

Trafic de convor. - On appelle trafic de con- 
voi un trafic qui possède la propriété de stabilité, et qui en outre est sans dépasscments (donc satisfait à l'hypothèse d'une vitesse localisée).

MÉthode de la RELATION FluX-densité. - Le principal travail fondé sur la méthode fluxdensité est [1], qui traite le problème suivant :

Soit $\mathrm{T}_{0}$ un trafic stationnaire, correspondant à des valeurs constantes $\rho_{0}$ et $\varphi_{0}$ de $\rho$ et $\varphi$, satisfaisant naturellement à :

$$
\varphi_{0}=\mathbf{G}\left(p_{0}\right)
$$

Soit à déterminer le trafic T qui s'établit à partir de $\mathrm{T}_{0}$, si $\mathrm{T}_{0}$ subit une petite perturbation; mathematiquement, tout revient, dans le cadre d'une approximation linéaire, à déterminer les ondes sinusoïdales (prises sous forme complexe) du type :

$$
\begin{aligned}
& \rho=p_{0}+p_{1} e^{i \omega[t-(x / v)]} \\
& \varphi=\varphi_{0}+\varphi_{1} e^{i \omega[t-(x / v)]}
\end{aligned}
$$

où $\rho_{1}$ et $\varphi_{1}$ sont des constantes, qui satisfont identiquement à $(1,2)$ et $(2,1)$.

Je désigne par $\dot{G}(p)$ la dérivée

$$
(d \mathrm{G} / d \rho)=(d \varphi / d \rho)
$$

de $G$ par rapport à $\rho$, pour la valeur $\rho$ de la variable.

On trouve immédiatement que :

$$
v=\mathrm{G}\left(\mathrm{p}_{0}\right)
$$

où $v$ n'est autre que la vitesse de propagation de l'onde; on note que $v$ est indépendante de $\omega .(2,8)$ est indépendante de l'hypothèse d'une vitesse localisée.

Dans le cadre de la même approximation linéaire, il est alors facile d'examiner des questions telles que les suivantes:

$1^{\circ} \varepsilon$ étant un nombre positif petit, on considère un trafic $T$ qui, à l'instant initial $t=0$, a la structure suivante : pour $x>\varepsilon$, T est approximativement stationnaire, avec des valeurs constantes $\rho_{1}$ et $\varphi_{1}=\mathrm{G}\left(p_{1}\right)$ de $\rho$ et $\varphi$; pour $x<-\varepsilon$, $\mathrm{T}$ est approximativement stationnaire avec des valeurs constantes $\rho_{2}$ et $\varphi_{2}=\mathrm{G}\left(\rho_{2}\right)$ de $\rho$ et $\varphi, \rho_{z}$ étant $<\rho_{1}$; l'intervalle $(-\varepsilon,+\varepsilon)$ est une petite zone de transition; quelle est l'évolution de $T$ pour $t>0$ ? On constate que la petite zone de transition se propage, à peu près à la manière d'une onde de choc, avec une vitesse de propagation approximativement égale à :

$$
\frac{1}{2}\left[\dot{G}\left(\rho_{1}\right)+\dot{G}\left(\rho_{2}\right)\right] \text {. }
$$

$2^{\circ}$ Le trafic étudié $\mathrm{T}_{0}$ est un trafic stationnaire, de densité constante $\rho_{0}$ et de flux constant $\varphi_{0}$, avec $\varphi_{0}=\mathrm{G}\left(\rho_{0}\right)$, alimenté par une «source» de véhicules dont le débit a en principe la valeur constante $\mathrm{D}_{0}$; toutefois, pendant un bref inter- valle de temps d'amplitude $\Delta t$, le débit de la somme atteint une valeur $D_{0}+\Delta D_{0}$ supérieure à $\mathrm{D}_{0}$, créant ainsi une «bosse » de trafic; comment se propage cette bosse?

Il s'agit encore d'un problème d'onde de choc; la «bosse » tend à disparaître.

$3^{\circ}$ La voie, uniforme dans son ensemble, présente sur une longueur $L$ un rétrécissement peu accusé, c'est-à-dire un goulot d'étranglement; alors la fonction $G$ n'est pas la même pour la voie dans son ensemble, et pour le troncon de longueur $\Delta L$ constituant le goulot. L'étude est encore celle d'une propagation d'onde de chocs.

[1] indique même comment la méthode cidessus peut apporter d'utiles indications dans l'étude d'un croisement.

Dans [1], les auteurs ont extrêmement simplifié l'appareil mathématique; mais ils ont donné de celui-ci une discussion beaucoup plus complète dans l'article suivant :

M. J. Lighthill et G. B. Wrtham. - On kinematic waves, Part. I, paru dans le même tome de la même revue que [1].

L'article [2] est similaire à [1], avec la différence que [2] adopte pour $G(p)$ la forme analytique déterminée suivante :

$$
\Phi=\mathrm{G}(\rho)=\varphi_{m} \cdot 4 \frac{\rho}{\rho_{c}}\left(1-\frac{\rho}{\rho_{c}}\right),
$$

où les constantes $\rho_{c}, \varphi_{m}$ ont les significations précisées ci-dessus. P. L. Richards, auteur de [2], justifie le choix particulier $(2,9)$ de $G(p)$ en disant que c'est une forme simple (ce qui est vrai), et expérimentalement «aussi justifiée qu'une autre »; il est aujourd'hui reconnu que $(2,9)$ s'adapte mal en général aux trafics réels; mais dans certains cas, $(2,9)$ peut constituer une approximation simple acceptable de la fonction $G$ véritable.

Quoi qu'il en soit, avec $(2,9)$ et en posant :

$(1,2)$ s'écrit :

$$
\rho=\rho_{c} \bar{\rho}
$$

$$
\frac{\partial \bar{\rho}}{\partial t}+4 \frac{\varphi_{m}}{\rho_{c}}(1-2 \bar{\rho}) \frac{\partial \bar{\rho}}{\partial x}=0
$$

dont la solution la plus générale est :

$$
\bar{p}=f\left[x-4 \frac{\varphi_{m}}{\rho_{c}}(1-2 \bar{\rho}) t\right],
$$

où $f$ est une fonction arbitraire. De là, il est facile de tirer des résultats, qui ne sont en définitive que des cas particuliers de ceux de [1]; à noter que l'auteur, P. I. Richards, de [2] :

$1^{\circ}$ remarque justement que le principe de sa méthode ne dépend pas du choix particulier $(2,9)$ de $\mathrm{G}$; 
$2^{\circ}$ signale explicitement qu'il fait l'hypothèse d'une vitesse localisée, et que cela implique l'bypothèse d'un trafic sans dépassements;

$3^{\circ}$ s'intéresse particulièrement aux problèmes posés par les croisements.

Dans [4], M. J. Lighthill reprend le point de vue et la méthode de [1], mais il propose de la compléter - et de la compliquer, de la facon suivante :

$1^{\circ}$ Chaque conducteur $\mathrm{C}$ agit essentiellement en fonction du comportement des véhicules qui sont immédiatement devant lui - - tout au moins Iorsque ceux-ci tendent à ralentir, et par conséquent à créer en ce qui les concerne une densité $\rho^{\prime}$ plus forte que celle $\rho$ qui concerne $C$ luimême : $\mathrm{C}$ règle alors son action sur $\rho^{\prime}>p$; l'auteur propose de tenir compte de cet effet en ajoutant au deuxième membre de $(2,1)$ un terme de la forme :

$$
\mathrm{D} \frac{\partial^{2} \varphi}{\partial x^{2}}
$$

$2^{\circ}$ Un conducteur a une certaine inertie, c'està-dire ne réagit pas instantanément et a besoin d'un certain délai pour accélérer ou freiner; l'auteur propose d'en tenir compte en ajoutant au deuxième membre de $(2,1)$ un terme de la forme :

$$
\mathrm{T} \frac{\partial^{2} \varphi}{\partial t^{2}}
$$

Ainsi, l'équation $(2,1)$ s'écrirait finalement :

$$
\varphi=\mathrm{G}(\rho)+\mathrm{D} \frac{\partial^{2} \varphi}{\partial x^{2}}+\mathrm{T} \frac{\partial^{2} \varphi}{\partial t^{2}} ;
$$

bien qu'il ne le dise pas explicitement, il semble que, dans l'idée de l'auteur, les coefficients $D$ et T. seraient des constantes. L'équation $(2,10)$ est d'un type relativement complexe, mais qui a justement déjà été rencontré et étudié en hydrodynamique.

\section{3. - ETUDE SIMULTANEE D'UN TRAFIC ET DU TRAFIC INVERSE}

Les travaux précédents font abstraction du trafic inverse du trafic étudié; c'est-à-dire qu'ils font l'hypothèse que le trafic inverse, s'il existe, est sans interaction sur le trafic étudié. Cette hypothèse n'est pas toujour's satisfaite dans la réalité, et [6] s'est proposé de faire l'étude simultanée d'un trafic $\mathrm{T}$ et de son trafic inverse $\mathrm{U}$, sans exclure les interactions éventuelles de ces deux trafics, mais en reprenant les principes de [1].

Soient $\rho$ et $\varphi$, resp. $\sigma$ et $\psi$ les densités et flux respectivement de $\mathrm{T}$ et $\mathrm{U}$, avec naturellement : $\varphi \geqq 0, \psi \leqq 0$. Sans admettre nécessairement l'hypothèse de vitesses localisées, posons selon $(1,1)$ :

$$
\varphi=\rho u, \quad u \geqq 0 ; \quad \psi=\sigma v, \quad v \leqq 0 .
$$

I.es équations de continuité pour $\mathrm{T}$ et $\mathrm{U}$ s'écrivent respectivement :

$$
\frac{\partial \rho}{\partial t}+\frac{\partial \varphi}{\partial x}=0, \quad \frac{\partial \sigma}{\partial t}+\frac{\partial \psi}{\partial x}=0 .
$$

L'analogue de $(2,1)$ s'exprime ainsi : $u$ et $v$ sont des fonctions déterminces $f(\rho, \sigma)$ et $g(\rho, \sigma)$ de $\rho$ et $\sigma$ :

$$
u=f(\rho, \sigma), \quad v=g(\rho, \sigma) .
$$

[6] fait sur $f$ et $g$ les hypothèses suivantes :

a) $f$ est une fonction monotone décroissante de $\rho$ d'une part, de $\sigma$ d'autre part; $g$ est une fonction monotone croissante de $\rho$ d'une part, de $\sigma$ d'autre part.

b) pour tout $\sigma$, il y a une valeur $\rho_{c}(\sigma)$ de $\rho$ telle que : $f\left[p_{o}(\sigma), \sigma\right]=0$; pour tout $\rho$, il y a une valeur $\sigma_{c}(\rho)$ de $\sigma$ telle que $g\left[\rho, \sigma_{c}(\rho)\right]=0$.

c) $f(\rho, \sigma)=-g(\sigma, \rho)$ (symétrie du trafic $\mathrm{T}$ et de son inverse $U)$.

Pour intégrer, compte tenu de $(3,3)$, les équations aux dérivées partielles $(3,2)$, on peut rechercher leurs caractéristiques dans le plan $(t, x)$. On peut aussi échanger les rôles de $(t, x)$ et de $(\rho, \sigma)$ c'est-à-dire prendre $(\rho, \sigma)$ comme variables et $(t, x)$ comme inconnues; les équations $(3,2)$ sont alors remplacées par :

$$
\begin{aligned}
& \frac{\partial x}{\partial \rho}-\left(u+\rho \frac{\partial u}{\partial \rho}\right) \frac{\partial t}{\partial \sigma}+\rho \frac{\partial u}{\partial \sigma} \frac{\partial l}{\partial \rho}=0, \\
& \frac{\partial x}{\partial \sigma}-\left(v+\sigma \frac{\partial v}{\partial \sigma}\right) \frac{\partial t}{\partial \rho}+\sigma \frac{\partial v}{\partial \rho} \frac{\partial t}{\partial \sigma}=0 .
\end{aligned}
$$

On peut alors chercher les caractéristiques, dans le plan $(\rho, \sigma)$, des équations $(3,4)$, qui ont l'avantage d'être linéaires par rapport aux inconnues $t$ et $x$.

[6] met en évidence le fait suivant : Ies équations sont des équations de propagation, et devraient donc être de type hyperbolique : en fait, pour un certain domaine $(\mathcal{D})$ du plan $(\rho, \sigma)$, ces écuations se révèlent être du type elliptique; les auteurs ne donnent pas d'explication définitive pour ce paradoxe, mais suggèrent qu'il provient peut-être de ce que les relations flux-densité $(3,3)$ sont inadmissibles en cas de régime rapidement variable.

A titre d'exemple, [6] traite en détail le cas où $f$ et $g$ sont linéaires (ce qui est l'extension au problème actuel du cas traité par [2] pour un trafic indépendant du trafic inverse); les calculs sont alors naturellement assez élémentaires; l'étude porte principalement sur la propagation des perturbations, la stabilité, etc. 


\section{4. - DETERMINATION DE LA RELATION FLUX-DENSITE}

Revenant au cas d'un trafic indépendant du trafic inverse, le travail [5] de $H$. Greenberg se distingue des précédents: Greenberg a pensé qu'on pouvait compléter l'équation de continuité par une équation dynamique au lieu d'une relation flux-densité. Pour cela, admettant l'hypothèse d'une vitesse localisée, il introduit une notion de pression; il admet que tout véhicule situé à l'instant $t$ à labscisse $x$ subit wne pression $p=p(t, x)$, qui represente et résume l'action sur lui de tout l'univers extérieur; moyennant quoi l'équation de la dynamique s'écrit naturellement:

$$
\frac{d u}{d t}=\frac{1}{\rho} \frac{\partial p}{\partial x}
$$

Il importe d'abord de bien apprecier ce qu'implique l'introduction d'une telle pression: d'ahord, dans l'action sur un véhicule de l'univers extériem, [5] néglige tout chanp de force tel que pesanteur, etc: et admet que cette action est purement locale, autrement dit, postule que, parmi les réhicules du trafic, les senls à influer notablement sur un péhicule donné quelconque sont ses roisins immediats. Il est clair que dans la réalité un conducteur penut etre influencé par le comportement de Tethicules qui ne sont pas contigus all sien. Neammoins, le postulat de H. Greenberg est admissible, an moins en première approximation, poldr la jupart des trafics de convoì assez denses.

Mais les équations $(1,2)$ et $(4,1)$, ne suffisent encore pas à déterminer mathématiquement le trafic, puisque l'équation supplementaire $-(4,1)$ comporte une inconnue, $p$, supplémentaire elle aussi. Pour obtenir ane équation de plus, H. Greenberg fait l'hypothèse que $p$ est une fonction déteminée:

$$
p=-\psi(p)
$$

de peul, la relation $(4,2)$ étant valable de facon absolue et en toutes circonstances. Quelque argument qu'on puisse donner en faveur de cette hypothèse, on ne peut nier son caractère arbitraire; le principal reproche que je lui ferais est que, si une relation telle que $(4,2)$ est plausible en régime stationnaire, elle est inadmissible en régime rapidement variable.

Quoi qu'il en soit, en admettant $(4,2)$, le système (S) des équations $(1,2),(4,1)$ et $(4,2)$ détermine le trafic; $H$. Greenherg s'est alors posé la question suivante : quelle relation flux-densité :

$$
\psi=G(p)
$$

est impliquée, en régime stationnaire tout au moins, par le systeme (S)? Un calcul simple montre que le systeme (S) implique la relation flux-densité suivante:

$$
\varphi=\rho . \int_{\rho}^{\rho_{0}} \frac{1}{x} \sqrt{\frac{d}{d x} \psi(x)} d x
$$

$\mathrm{Or}$, et c'est là qu'il voulait en venir, H. Greenberg estime que pour les trafics de convoi assez denses, l'expérience prouve que la fonction G est de la forme;

$$
G(\rho)=u_{m} \rho \log \frac{p_{c}}{\rho},
$$

où $u_{m}$ est une constante, qui n'est autre que la valeur de $u$ correspondant aú flux maximal $\varphi_{m}$. Pour que $(4,3)$ soit de la forme $(4,4)$, il faut et il suffit, compte tenu de ce que nécessairement $\psi(0)=0$, que :

$$
\psi(p)=u_{m}^{2} p,
$$

ce qui procure une détermination indirecte de la fonction $\psi$ dont l'existence a été postulée.

Je ne crois pas utile d'analyser plus longuement ce travail [5] de $H$. Greenberg, que j'ai d'ailleurs déjà exposé et discuté plus en détail ailleurs (Cf. R. Fortet [1 bis]).

\section{5. - CONCLUSIONS}

Les travaux sur le trafic des véhicules, s'inspirant de la Mécaniques des fluides, ont rendu des services; mais, obligé de pallier l'absence de l'équation dynamique et de l'équation d'état par un recours à une loi empirique, et ce dans des conditions un peu contestables, ils fournissent des modèles parfois satisfaisants comme modèles descriptifs, ils ne fournissent en aucun cas une théorie explicative. En outre, leur portée est limitée, pratiquement, aux trafics de conroi; pour ces trafics de convoi justement, il existe maintenant une théorie directe beaucoup plus solide et féconde.

Pour élargir la portée des méthodes inspirées par la Mécanique des fluides, il faudrait en particulier se libérer de l'hypothèse d'une vitesse localisée, ce qui me semble parfaitement possible, puisqu'on a pu le faire dans l'élude du mouvement des électrons en interaction mutuelle dans un tube électronique. Mais alors, plus qu'avec la Mécanique classique des lluides continus, l'analogie sera avec une sorte de Mécanique statistique; mais e'est bien de la Mécanique statislique que cherchent à s'inspirer certains travaux récents, en particulier de I. Prigogine (Cf. par exemple 1. Prigogine, R. Herman, et R. Anderson [1 ter $]$ ).

\section{Bibliographie}

1 bis] R. Forter. - Théorie du trafic de convoï; Rev. Fr. de Rech. Oper., 6, 1962, p. 337.

[1 ter] 1. Prigogine, R. Herman et R. ANderson, - Further developments in the Boltzmamm-like approach of traffe flow; communication a $I^{\text {t }}$ Symposiam Int. sur la Thẻorie du trafic, Londres, 1963. 


\section{I S C U S S I O N}

Président : M. Chapouthien

M. Ie Président remercie M. Ie Professeur Fonter de son exposé qui se termine sur un horizon un peu hydraulique, alors que les hydrauliciens revendiquent aussi un peu la mécanique statistique qui est très à l'honneur, notamment pour l'étude de la turbulence.

M. le Professeur Bonnin s'exprime en ces termes:

«Je n'ai pas tellement l'intention de discuter la communication de M. le professeur Forter, mais plutôt d'apporter les excuses des hydraulicjens, parce qu'il y a ici un échantillon assez représentatif d'une population d'hydrauliciens, ou d'hydrotechniciens, ou de mécaniciens des fluides et il y a en même temps - je ne crois pas trop m'avancer en l'affirmant - un échantillon très représentatif d'une population d'automobilistes.

"Je pense qu'il nous est arrivé à tous de nous poser des problèmes de circulation automobile, d'en être spectateurs ou observateurs, parfois même forcés; il mous est arrivé d'être dans le cas où $p=p_{\theta}$, et quelquefois pendant un temps trop important.

"On peut se demander pourquoi tous ces hydrauliciens, dont je suis d'ailleurs, ne se sont pas posé la question et ont attendu que M. le Professeur Fonter vienne lui-même nous exposer le résultat de recherches sur la question et des rapports entre notre mécanique des fluides et la mécanique des automobiles.

\& Je voudrais, en quelque sorte, les excuser; je pense que personne d'entre nous n'osait aborder la question. Beaucoup ont essayé, mais n'osaient traiter le sujet, parce que, s'il y a quelques analogies avec la mécanique des fluides, il y a beaucoup de différences. Je ne prétends pas, bien entendu, énumérer toutes ces différences, mais je veux vous en donner quelques-unes, que j'ai classées en deux grandes catégories : différences d'ordre microscopique et différences d'ordre macroscopique.

«Comme différence d'ordre microscopique, je dirai d'abord que les molécules que nous avons à considérer - les automobiles en ce cas - sont beaucoup plus indépendantes que ne le sont les molécules dans Ia théorie cinétique des gaz (et, a fortiori, cncore plus dans le cas d'une colonne humaine soumise à un flux, comme dans l'exemple de $M$. le Président, auçuel cas il n'y a plus aucune différence entre les vitesses). Elles sont très différentes et il arrive (tout le monde l'a vu) qu'une molécule au milieu du canal - disons milieu d'une rue - marche systématiquement à une vitesse beaucoup plus faible et qu'il est impossible de la faire avancer plus vite.

«De plus, ces molécules ont la propriété d'avoir leur vikesse orientée à peu près toujours dans la même direction, avec des fluctuations assez faibles dans les directions transversales. C'est une différence très sensible avec la théorie cinétique des gaz, où les vitesses de fluctuations dues à la température sont à peu près isotropes.

«Pourquoi la vitesse qui se surajoute devient-elle prépondérante? Parce que, si nous voulons poursuivie l'analogie avec une théorie cinétique dc molécules en mouvement fluide, nous sommes à des vitesses très largement hypersoniques. Iá vitesse du déplacement devient prépondérante et les vitesses transversales a peu près véglìgeables.

«En ce qui concelne la dynamique, comme il a été dit tout à l'heure, il $y$ a une différence d'ordre psychologique, ce qui donne une bien plus grande dispersion que pour les molécules qui sont tout de méme assez dociles aux lois de la statistique.
«Il y a un très gros écueil, et lik, je crois que l'on louche aussi à la notion de pression. Ici, je pense que je vais discuter un peu plus directement la eommunication de $M$. le Professeur Fonter. Je crois qu'il n'y a pas, dans le schema actucl, de prineipe de laction el de la réaction. Autrement dit, lorsque le vébicule cou la molécule) qui est devant oblige celui (ou celle) qui se trouve derrice a avoir une action par un effet de pression dù à sa trop grande proximité ou a sa vilesse, la proximité étant $\rho$ naturellement, en revanche, ete molécule (ce véhicule) ne subit pas d'action de la part de celui qui est derrière, saur, bien entendu, s'il y a collision, ec qui arrive parfois... Il $y$ a la une tres grande différence entre les deux systèmes.

«De plus, les molécules sont orientées; elles ont des dimensions longitudinales supérieures aux dimensions transversales; ce n'est pas tres grave, seulement lorsque lon a des molécules de gaz diatomique, elles n'ont pas de préférence d'orientation.»

"Vous voyez qu'on s'éloigne assez progressivement de la théorie cinćtique: si l'on se plaçail au point de vue de la théorie cinétique dans un cas extrêmement particulier, il faudrait revoir la facon dont on pournat envisager de le traiter.

\&Il y a encore un autre élément: les trajectoires qui sont suivies par ces molécules sont in peu prés confondues et distinctes les whes des autres transversalement, avec des ecarts entre trajectoires qui sont quantifiés pour un écoulement donné on a peu prés. Donc, on se trouve encore aves une contrainte sujplémentaire, que l'on ne rencontre pas habituellement dans les écoulements de gaz.

«Si l'on veut parler maintenant din point de vue macroscopique, on soufrisa certainement wne petile difficulté : le macroscopicue n'est peut-être pas toul a fait légitime, mème sur une artère à $\tilde{5}, 6$ ou 10 voies de circulation, car ee n'est peut-être pas encore la loi des frands nombres qui va jouer. Ce n'est peut-itre pas lit que l'on verra la plus grande différenee, mais il faudma en tenir" compte.

«L'équation d'état est assez difficile à assimiler औ cause de ce manque de principe d'action et de réaction.

"On arrive au point suivant: si l'on veut juger sur les conséquences, il $y$ a des différences fondamentales au point de vue de la pression et de la réaction.

"Je peux citer M. Chapouthen, qui avait, de façon très imagéc, montré que Venturi s'est trompé quand il a voulu établir son appareil. Venturi cherchait une augmentation de pression; nous avors tous demontré qu'il y aura une diminution de pression pour un fluide comportant un trés grand nombre de molécules en mon. vement; or, nous savons fue, s'jl y a un convergent dans une vole de circulation automobile, cela se traduira bien par une augmentation de pression.

\& C'est intéressant, parce que nous pouvons penser que, si nous nous trouvons dans un schema moleculaire comparable au schema de circulation automobile, nous pouvons nous attendre à donner raison alu premier raisonnement de Venturi.

\&Il y a d'autres analogies. Je ne sait pas s'il faut considérer qu'il y a, pour ce schéma moléculaire, viscom sité ou non, en tout cas pas au sens newtonien. Doituon considérer qu'il y a vitesse nulle à la paroi je ne pense pas, même s'il y a une couche moléculaire de moléculesventouses le long d'un trottoir; je crois plutot le contraire; il arrive que la couche immediatement voisine 
soit préférentielle pour l'écoulement. On ne peut pas non plus parler d'efforts entre couches voisines proportionnels à leur écart de vitesse; c'est certainement quelque chose de très différent.

«En résumé, si nous voulions faire l'analogie en mécanique des fluides, nons aurions besoin d'étudier un fluide qui serait constitué, bien entendu, de molécules non sphériques, mais orientées; je pense d'ailleurs qu'elles sont orientées d'elles-mêmes, si l'on alteint les conditions suivantes': ces molécules seraient à une très grande vitesse par rapport à leur agitation thermique, c'est-à-dire un écoulement très hautement supersonique, beaucoup plus qu'on ne sait le faire actuellement, avec des dimensions transversales de l'écoulement comparables au libre parcours moyen, lequel est très faible, car, comme ces molécules sont très voisines les unes des autres, lo volume occupé est comparable; il est un petit nombre de fois plus grand que le volume de gaz; nous sommes donc, de plus, à très haute pression.

«Voici le probleme devant lequel nous nous trouvons, nous, mécaniciens des fluides, si nous voulons essayer de résoudre avec nos moyens les problèmes de circulation automobile.

«Que ferions-nous si nous voulions résoudre le problème de l'écoulement d'un fluide à des pressions extrômement élevées, des vitesses extrêmement élevées, et en considérant des dimensions transversales égales seulement à l'épaisseur de quelques molécules? Je pense que nous ferions une étude en similitude. Quel gente pouvons-nous essayer, tout au moins sur un modèle qui cherchera à représenter les choses? D'abord, on va adopter une échelle très grande. On voudra peüt-ètre donner aux molécules une possibilité d'expression personnelle : on va alors fournir à chaque particule un opérateud indépendant, qui sera chargé de regarder ce qui se passe autour et de travailler suivant son carnctère propre. Pour rendre lopérateur bien liè à la particule, il faudra donner à celle-ci une dimension de l'ordre de $4 \mathrm{~m}$ sur $1,50 \mathrm{~m}$; pour qu'elle soit mobile, on lui donnera des roues et peut-être un moteur, et là, je crois que nous rentrons exactement dans le cas que vous avez traitè. 》

M. Fonter indique qu'étant donné deux véhicules successifs, le second subit l'action du premier et l'inverse n'a pratiquement jamais lieu. On peut donc considérer essentiellement le mouvement des véhicules qui sont en tête dans une suite ordonnée de véhicules : le mouvement du deuxième véhicule résultera du premier, celui du troisième résultera du second, etc.

C'est à peu près sur ce principe que reposent les études de trafic de convoi acluelles. Si l'ordre des véhicules ne reste pas invariable, il $y$ a une complication qui n'a pas été vraiment étudiée. Il faut savoir tenir compte de ce changement d'ordre.

M. LaRras indique que la vitesse de propagation des perturbations est égale à $d G / d_{\rho}$. Or $d G / d_{\rho}$ est nul au voisinage du débit optimal de la route (fig. 2-1 du mémoire). On arriverait done à ce résultat inattendu qu'aucune perturbation ne se propagerait aux environs du débit optimal. Est-ce bien exact?

M. Forter répond que malheureusement certaines personnes disent que la zone avoisinant le maximum est loin d'avoir l'allure si satisfaisante de sommet de parabole que les théoriciens nous proposent.

En réalité, «ce sommet» est à peu près l'emplacement d'un changement de régime. A gauche, sur la figure, on a des densités pas très grandes auxquelles ces théories ne prétendent guère s'appliquer et où les conducteurs sont assez libres de faire ce qu'ils veulent, puisqu'il n'y a pas beaucoup de véhicules pour les gêner : bref, c'est un schéma stochastique qui pourrait rendre compte de ce qui se passe.

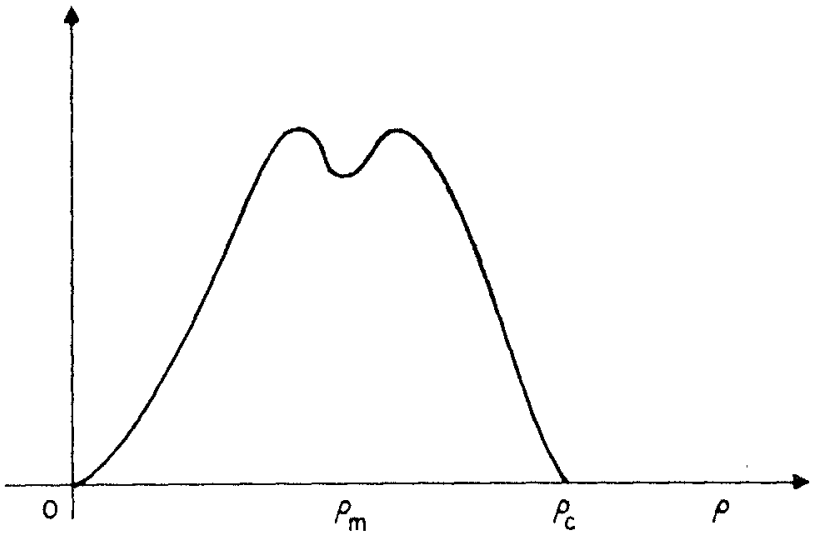

Si l'on approche des densités plus fortes, peu à peu, les conducteurs sont obligés de se discipliner et modifient sourent leur façon de conduire à ce moment-là; certains prétendent qu'il y a, en fait, un premier maxinum, puis un second, la zone intermédiaire étant très trouble.

D'autres disent que tout ceci est une vue de l'esprit, que e'est très difficile à observer et que ce n'est pas la peine de s'en préoccuper.

Par contre, le fait que $v$ soit négatif d'un côté et positif de l'autre, si l'on n'est pas trop près du maximum, est très net; Lighthill s'en sert systématiquement.

M, le Président pense qu'il peut y avoir deux l'égimes permanents séparés par une petite transition. II $y$ a propagation d'une onde de choc comme on l'a quand on veut représenter la fermeture instantanée d'une colonne liquide par un robinet, avec une variation brutale de pression (méthode Bergeron).

M. Fonter indique que, sur d'autres sortes de schémas, les périodes transitoires ont fait l'objet d'études sérieuses; elles s'associent forcément aux études des fluides.

M. Wegner mentionne que des travaux étudiés par M. Fontex postulent tous un réginue quasi stationnaire de la circulation, ce qui est nature]]cment gênant pour un tel phénomène. Les essais d'un modèle mathématique de circulation urbaine, faits par la SO.GR.E.A.H., permettent de dire que la difficulté peut itre tournée en partageant les voies en un certain nombre de tronçons élémentaires. On suppose alors vitesse et densité constantes dans chaque troncon avec une bonne approximation, l'état de la circulation ne variant que d'un tronçon à l'autre. Par exemple, si «dans la nature» la mise au rouge d'un fieu de croisement provoque en amont une certaine «courbe de remous» en densité, on la retrouve sous forme d'un escalier d'autant plus proche que les tronçons élémentaires sont plus petits.

Cette méthode n'est évidemment possible qu'en utilisant un ordinateur électronique puissant, capable de garder en mémoine le grand nombre de tronçons représentant les voies.

M. Forter dit qu'il y a, dans Lighthill, une étude qui se rapproche de l'idée d'une voie en principe uniforme mais avec, $\dot{a}$ un certain endroit, un goulot d'étranglement. Il y a un régime stationnaire avant et un autre après. Lighthill supposait, pour la zone du goulot d'étranglement assez courte, que la fonction $G$ n'était pas la même. Il $y$ avait un $\ll$ $G »$ spécial pour cette zone. Il étudiait le raccordement entre les deux parties stationnaires avec ce passage intermédiaire.

M. le Président remercie à nouveau $M$. le Professeur Fonter des aperçus très intéressants qu'il a donnés. 